\title{
Unusual location of syphilitic chancre: case report
}

\author{
POMPEO DONOFRIO \\ From the Department of Dermatology, Second School of Medicine, University of Naples, Naples, Italy
}

SUMMARY A case of secondary syphilis in which the patients still had the primary chancre is reported. The unusual clinical feature was the location of the syphilitic chancre on the arm.

\section{Introduction}

Extragenital primary syphilis ranges in incidence from $2 \%$ to $10 \%^{1}$ and usually results from contact with genital or extragenital lesions during sexual foreplay. Syphilitic chancre located on the fingers ${ }^{1}$ and hands $^{2}$ has been reported rarely. In 1983 I saw a patient with primary syphilis on the arm and, in view of the rare location, present the case history.

\section{Case report}

A married man aged 50 first attended this dermatology clinic in May 1983 as he had noted a nonpruritic generalised papular skin rash. He complained of having had an ulcerative lesion on his right arm for six to eight weeks. He had no history of puncture wounds or bites and had had no recent antibiotic treatment.

Examination showed a symmetrically distributed papular rash on the face, neck, trunk, legs, and arms. The lesions were brownish-red and were 0.5 to $1 \mathrm{~cm}$ in diameter. His palms, soles, mouth, and ano-genital region were not affected. On the right arm (fig 1) there was a papular lesion with a smooth depressed surface and raised indurated borders. The right axillary lymph nodes were enlarged, firm, rubbery, and not tender. There was generalised mild lymph node enlargement. Microscopic examination of a skin biopsy specimen from the chancriform lesion on the right arm showed (fig 2) a dense infiltrate composed of lymphocytes and plasma cells surrounding the blood vessels in the dermis. Serological tests for syphilis (Venereal Disease Research Laboratory (VDRL) test, Treponema pallidum haemagglutination assay (TPHA), and fluorescent treponemal antibody absorbed (FTAABS test) all gave positive results. The VDRL slide test was reactive at a titre of $1 / 64$. His wife also gave positive results to serological tests for syphilis.

The patient was treated with benzathine penicillin

Address for reprints: Dr Pompeo Donofrio, Clinica Dermatologica, II Facolta di Medicina, Via S Pansini 5, 80131 Napoli, Italy

Accepted for publication 1 July 1985
1.2 MIU three times a week for 10 injections (a total dose of $12 \mathrm{MIU}$ ). There was no Jarisch-Herxheimer rection. One month after treatment (fig 3) the ulcerative lesion had healed leaving a thin atrophic scar and the rash had disappeared. The serum VDRL titre fell to $1 / 8$ and became negative within six months.

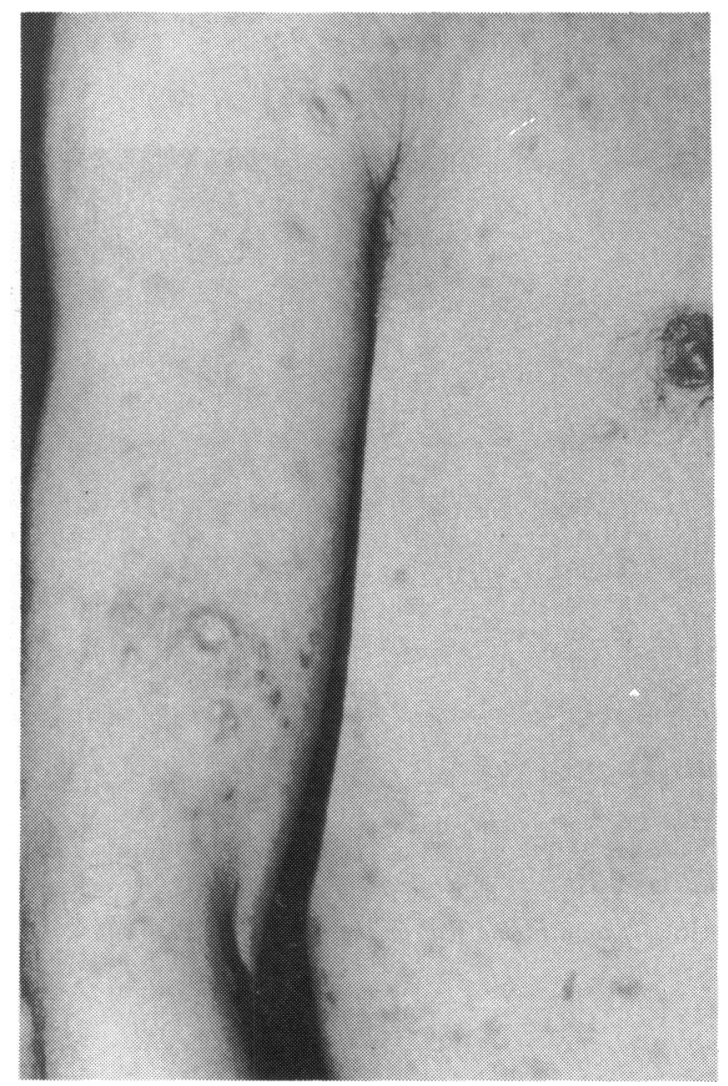

FIG I Brownish-red papules on trunk and right arm, ulcerative papular lesion on the arm. 

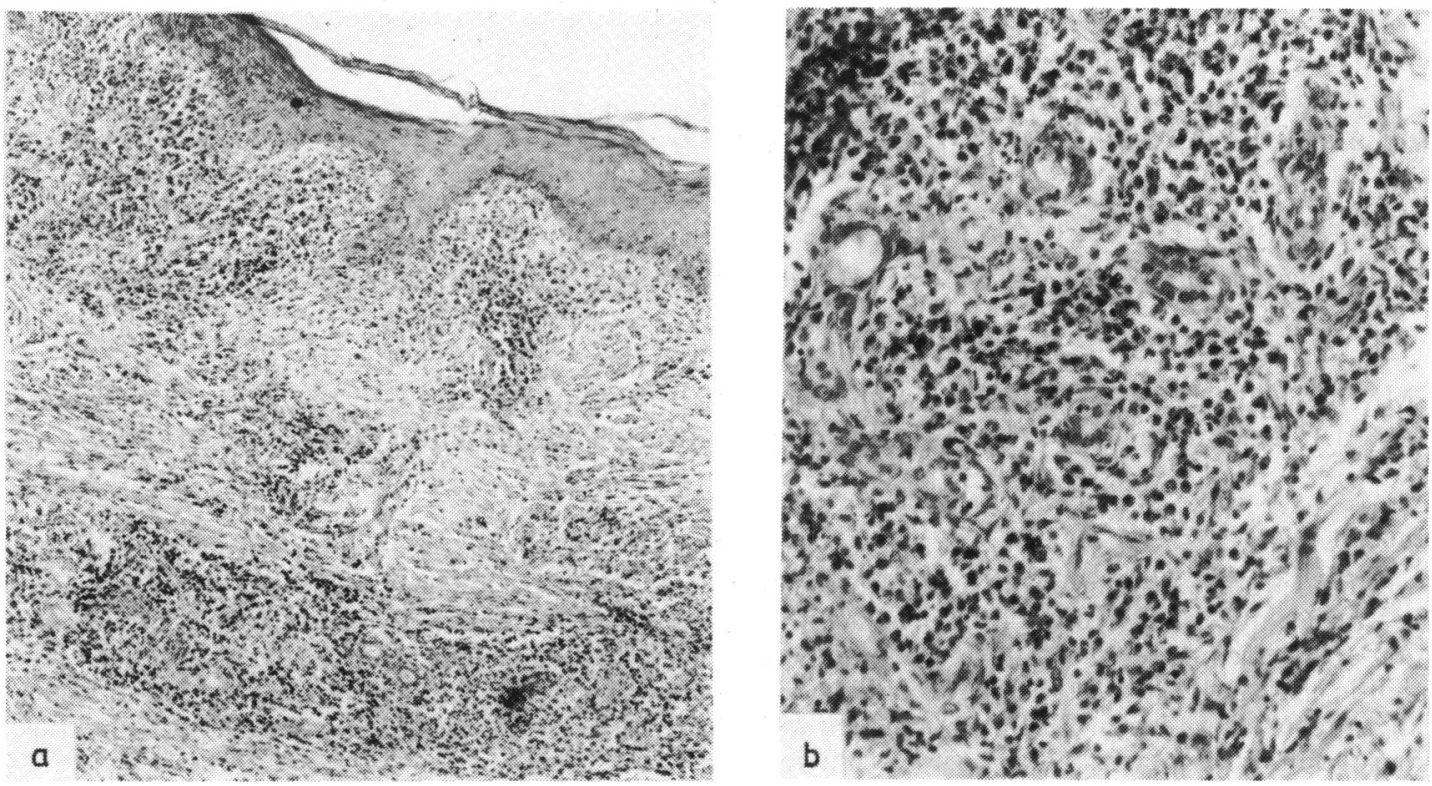

FIG 2 Dermis containing a dense lymphocyte and plasma cell infiltrate around the blood vessels (haematoxylin and eosin (a) (x 45 magnification) and (b) (x 207 magnification).

\section{Discussion}

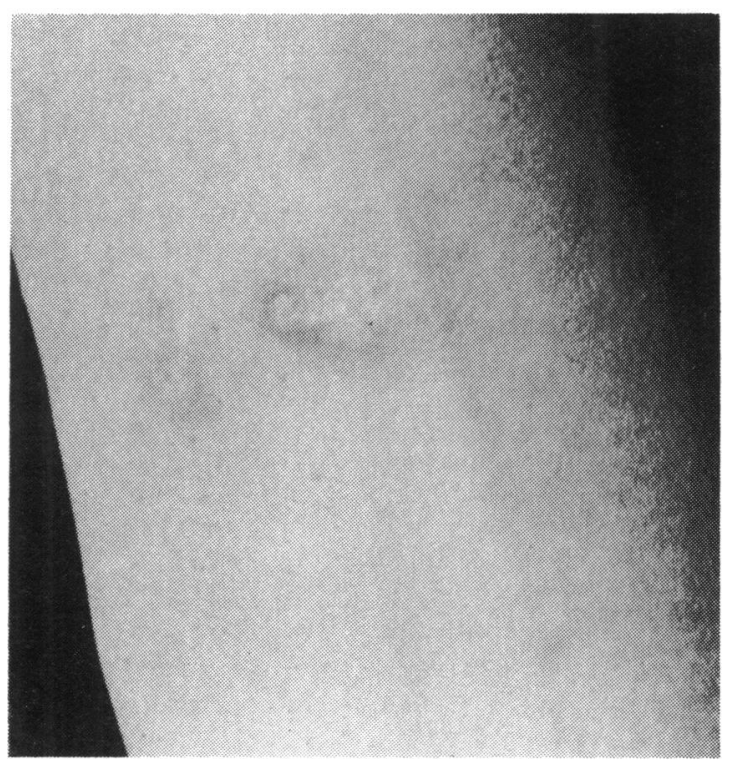

FIG 3 Thin atrophic scar on the arm after treatment.
Extragenital primary syphilis has been reported after puncture inoculation with infected syringes and tattooer's needles ${ }^{3}$ or a human bite. ${ }^{4}$ In our patient the mode of inoculation of the arm is difficult to explain. Moreover the clinical and histological features, the serological examinations, the effectiveness of antisyphilitic treatment, and the healing with a thin atrophic scar support the diagnosis of the chancre as being that of primary syphilis. These findings also exclude the diagnosis of a so called "chancriform papule" that represents only a sequela of secondary syphilis. ${ }^{5}$

\section{References}

1. Starzycki Z. Primary syphilis of the fingers. British Journal of Venereal Diseases 1983;59:169-71.

2. De Koning GAJ, Blog FB, Stolz E. A patient with primary syphilis of the hand. British Journal of Venereal diseases 1977;53:386-8.

3. Soendjojo A, Boedisantoso M, Ilias MI, Rahardjo D. Syphilis d'emblée due to blood transfusion: case report. British Journal of Venereal Diseases 1982;58:149-50.

4. Fiumara NJ, Exner JH. Primary syphilis following a human bite. Sex Transm Dis 1981;8:21-2.

5. Murugan S, Jeyasingh P, Prasad PVS. Chancriform papule: a case report. British Journal of Venereal Diseases 1983;59:402-3. 